\title{
偏轴磁控溅射法外延 $\mathrm{BiFeO}_{3}$ 薄膜的介电性能与阻变效应
}

\author{
宋建民 ${ }^{1,2}$, 代秀红 ${ }^{1}$, 梁杰通 ${ }^{1}$, 赵 䂞 ${ }^{1}$, 周 阳 ${ }^{1}$, \\ 葛大勇 ${ }^{1}$, 孟旭东 ${ }^{3}$, 刘保亭 1
}

(1. 河北大学 物理科学与技术学院, 保定 $071002 ; 2$. 河北农业大学 理学院, 保定 071001 ; 3. 河北北方学院 理学 院, 张家口 075000)

摘 要: 利用偏轴射频磁控溅射法, 在(001) $\mathrm{SrTiO}_{3}(\mathrm{STO})$ 单晶基片上制备了 $\mathrm{Pt} / \mathrm{BiFeO}_{3} / \mathrm{La}_{0.5} \mathrm{Sr}_{0.5} \mathrm{CoO}_{3} / \mathrm{STO}(\mathrm{Pt} / \mathrm{BFO} /$ $\mathrm{LSCO} / \mathrm{STO}$ ) 异质结电容器。研究了 $\mathrm{BiFeO}_{3}$ 薄膜的结构和物理性能。原子力显微镜( AFM) 和 X 射线衍射(XRD)分析 表明: BFO 薄膜结晶质量良好, 且为单相 $(001)$ 外延钙钛矿结构。介电性能测试结果发现: 在 $5 \mathrm{~V}$ 驱动电压下, $\mathrm{Pt} / \mathrm{BFO} / \mathrm{LSCO}$ 电容器呈现饱和的蝶形回线, 调谐率和介电损耗分别为 $14.1 \%$ 和 0.19 。此外, 阻变机制研究表明: 在 $0 \rightarrow 5 \rightarrow 0 \mathrm{~V}$ 正向电压和 $0 \rightarrow-5 \rightarrow 0 \mathrm{~V}$ 负向电压下, 阻变均为高阻向低阻转变规律, 呈现为铁电二极管的阻变开关行 为。通过 $I-V$ 曲线拟合, 得到 $0 \rightarrow 5 \rightarrow 0 \rightarrow-5 \mathrm{~V}$ 时阻变机制为空间电荷限制电流陷阱能级的填充和脱陷, 而 $-5 \rightarrow 0 \mathrm{~V}$ 时符合界面限制的 F-N 隧穿机制。

关 键 词: 铁酸铋薄膜; 介电常数; 阻变效应; 导电机制

中图分类号: O484 文献标识码: A

\section{Resistive Switching Effect and Dielectric Property of Epitaxial $\mathrm{BiFeO}_{3}$ Thin Films by Off-axis Magnetron Sputtering}

\author{
SONG Jian-Min ${ }^{1,2}$, DAI Xiu-Hong ${ }^{1}$, LIANG Jie-Tong ${ }^{1}$, ZHAO Lei ${ }^{1}$, \\ ZHOU Yang ${ }^{1}$, GE Da-Yong ${ }^{1}$, MENG Xu-Dong ${ }^{3}$, LIU Bao-Ting ${ }^{1}$
}

(1. College of Physics Science \& Technology, Hebei University, Baoding 071002, China; 2. College of Science, Agriculture University of Hebei, Baoding 071001, China; 3. College of Science, Hebei North University, Zhangjiakou 075000, China)

\begin{abstract}
The $\mathrm{Pt} / \mathrm{BiFeO}_{3} / \mathrm{La}_{0.5} \mathrm{Sr}_{0.5} \mathrm{CoO}_{3} / \mathrm{SrTiO}_{3}(\mathrm{Pt} / \mathrm{BFO} / \mathrm{LSCO} / \mathrm{STO})$ heterostructures were fabricated on (001) $\mathrm{SrTiO}_{3}$ substrate by off-axis RF magnetron sputtering, on which epitaxial $\mathrm{BiFeO}_{3}$ (BFO) thin films were suecessfully grown. The BFO thin film shows good crystal quality with (001) epitaxial growth as confirmed by the atomic force microscope (AFM) and X-ray diffraction (XRD). Pt/BFO/LSCO capacitor exhibits a saturated butterfly loop with tuning rate of $14.1 \%$ and dielectric loss of 0.19 at $5 \mathrm{~V}$ driving voltage. Moreover, the resistance changes from high value to low value with positive voltage $0 \rightarrow 5 \rightarrow 0 \mathrm{~V}$ and negative voltage $0 \rightarrow-5 \rightarrow 0 \mathrm{~V}$, which displays switch behavior of ferroelectric diode resistance-change. Based on the $I-V$ fitting curves, the resistance change mechanism fits the space charge limited current trap level when $0 \rightarrow 5 \rightarrow 0 \rightarrow-5 \mathrm{~V}$ and the interface limit F-N tunneling mechanism as $-5 \rightarrow 0 \mathrm{~V}$, respectively.
\end{abstract}

Key words: $\mathrm{BiFeO}_{3}$ thin films; dielectric constant; resistive effect; conduction mechanism

收稿日期：2017-12-20；收到修改稿日期：2018-02-11

基金项目：国家自然科学基金(11374086, 11474174); 河北省自然科学基金(E2014201188, E2014201063, A2018201168); 河 北省教育厅基金(2016058); 河北农业大学理工基金(ZD20160614)

National Natural Science Foundation of China (11374086, 11474174); Natural Science Foundation of Hebei Province (E2014201188, E2014201063, A2018201168); Foundation of Education Bureau of Hebei Province (2016058); Science and Technology Fund of Hebei Agriculture University (ZD 20160614)

作者简介: 宋建民(1976-), 男, 博士研究生. E-mail: sjm@hebau.edu.cn

通讯作者: 刘保亭, 教授. E-mail: btliu@hbu.edu.cn 
电致阻变效应随机存储器是极具前景的新一代 非易失性存储器, 具有高存储密度、低功耗、读写 速度快和结构简单等优点, 引起了广泛关注 ${ }^{[1-3]}$ 。在 许多钙钛矿结构氧化物如 $\mathrm{Ba}_{x} \mathrm{Sr}_{1-x} \mathrm{TiO}_{3}(\mathrm{BST})$ 、 $\mathrm{Pb}\left(\mathrm{Zr}_{x} \mathrm{Ti}_{1-x}\right) \mathrm{O}_{3}(\mathrm{PZT})$ 和 $\mathrm{BiFeO}_{3}(\mathrm{BFO})$ 中均发现了阻 变效应 ${ }^{[4-6]}$ 。铁酸铋 $\mathrm{BiFeO}_{3}(\mathrm{BFO})$ 是一种室温下兼具铁 电性和铁磁性的多铁性材料, 且具有较窄的禁带宽 度 $(2.67 \mathrm{eV})$, 为形成高低阻转变提供了更大的可能, 因此 BFO 成为近年研究的热门阻变材料之一 ${ }^{[7-8]}$ 。

目前, 国内外学者对新型非易失性 BFO 铁电阻 变存储器进行了较为广泛而深入的研究。 $\mathrm{Li}$ 课题组 ${ }^{[9]}$ 研究了 $\mathrm{Metal} / \mathrm{Bi}_{0.95} \mathrm{La}_{0.05} \mathrm{FeO}_{3} / \mathrm{Pt}$ 结构的阻变特性, 将阻变效应归因于导电细丝的形成与断裂。Zhu 等 ${ }^{[10]}$ 将 $\mathrm{Pt} / \mathrm{BFO} / \mathrm{SrRuO}_{3}$ 异质结中的阻变现象归因于陷阱 能级对电荷的俘获或释放。Park 课题组 ${ }^{[11]}$ 利用极化 调节 Schottky 势垒理论解释了 $\mathrm{BFO}$ 薄膜的阻变现 象。Yamada 等 ${ }^{[12]}$ 将 BFO 薄膜的阻变效应归因于异 质结的铁电隧穿。总而言之, $\mathrm{BFO}$ 薄膜的阻变效应 可归结为四种机制: 陷阱电荷的捕获与脱陷, 铁电 隧穿, 导电细丝的形成与断裂, 极化调节 Schottky 势垒。各种机制研究表明电极/氧化物界面在阻变反 转中起着至关重要的作用。不同接触电极和不同界 面都会使阻变特性不尽相同。然而, 根据器件表现 的阻变特性的不同, 其主导机制仍然未形成统一定 论。此外, 阻变存储器通常采用(上电极/介质/底电 极/基片)薄膜异质结构架结构 ${ }^{[13]}$, 可见, 阻变介质 材料、电极材料、薄膜取向及制备工艺也是影响阻 变存储器性能的因素。而在电极和介质层的制备过 程中, 薄膜的控制生长是最关键的环节。目前, 制 备 BFO 薄膜的方法主要包括溶胶一凝胶法 ${ }^{[14]}$ 、脉冲 激光沉积法 $(\mathrm{PLD})^{[15]}$ 和磁控溅射法(MS) $)^{[16]}$ 等。磁控 溅射法具有溅射工艺重复性好、薄膜质量高、与基 片结合较好、膜厚可控等诸多优点。然而, 此方法 沉积速率相对较低, 偏轴磁控溅射技术(OMS)恰好 可以弥补此缺点, 提高㳚射产额, 并且可以有效减 少电子的二次溅射, 使沉积出来的薄膜层具有非 常好的均匀性和致密性 ${ }^{[17-18]}$ 。因此 OMS 已成为沉 积高质量 BFO 薄膜及其它氧化物薄膜的主流工艺 之一。

截至目前, 虽然一些科研人员已对 BFO 薄膜阻 变效应进行了诸多研究, 但是在(001)STO 祄底上, 以 LSCO 作为底电极, $\mathrm{Pt}$ 作为上电极, 系统研究外延 $\mathrm{BFO}$ 薄膜的微观结构、介电性能和铁电阻变效应的 导电机理未见报道。本工作采用偏轴磁控溅射法, 在(001)STO 祄底上, 制备了 $\mathrm{Pt} / \mathrm{BFO} / \mathrm{LSCO}$ 异质结
阻变器件, 研究了 $5 \mathrm{~V}$ 电压作用下外延 $\mathrm{BFO}$ 薄膜的 介电性能及其阻变效应的产生机制。

\section{1 实验方法}

\section{1 样品制备}

采用射频磁控溅射法制备了 $\mathrm{Pt} / \mathrm{BFO} / \mathrm{LSCO} /$ STO 异质结，具体制备步骤如下:

(1)基片准备

将钛酸锶(001) $\mathrm{SrTiO}_{3}$ 单晶基片先后放入高纯 丙酮和无水乙醇中, 利用超声波清洗 $10 \mathrm{~min}$, 以此 去除基片表面的污染，然后迅速将基片放入磁控溅 射实验仪的真空腔中。

(2)偏轴射频磁控溅射法制备 LSCO 薄膜

利用机械泵和分子泵将放有基片的磁控溅射仪 腔体中的内背底真空度抽至 $2.0 \times 10^{-4} \mathrm{~Pa}$ 以下; 打开 流量计和进气阀使腔体充入高纯氩气和氧气，调节 流量计设置流量为 $100 \mathrm{sccm}, \mathrm{Ar}: \mathrm{O}_{2}$ 比例为 $3: 1$; 调节闸板阀, 使腔体中压强保持在 $1.4 \mathrm{~Pa}$; 调节射 频功率旋钮, 功率为 $50 \mathrm{~W}$; 保持靶衬间距为 $55 \mathrm{~mm}$, 且成 $45^{\circ}$ 角度, 沉积温度保持为 $550^{\circ} \mathrm{C}$; 为了去除靶 材表面的污染物, 高纯( $99.99 \%)$ LSCO 靶材预溅射 $20 \mathrm{~min}$, 正式溅射 $30 \mathrm{~min}, \mathrm{STO}$ 基片上外延生长厚度 约为 $40 \mathrm{~nm} \mathrm{LSCO}$ 薄膜。

(3)偏轴射频磁控溅射法制备 BFO 薄膜

利用与 LSCO 薄膜相同的沉积设备, 在 LSCO/ $\mathrm{STO}$ 异质结上外延生长约 $220 \mathrm{~nm}$ 厚的 $\mathrm{BFO}$ 薄膜。 为了避免 $\mathrm{Bi}$ 的挥发造成 $\mathrm{BFO}$ 薄膜偏析, 采用 $\mathrm{Bi}$ 过 量 $10 \mathrm{~mol} \%$ 的 $\mathrm{Bi}_{1.1} \mathrm{FeO}_{3}$ 靶材。磁控溅射沉积条件如 下: 沉积温度为 $625^{\circ} \mathrm{C}$; 腔体压强为 $2 \mathrm{~Pa}$; 气体流量 为 $100 \mathrm{sccm} ; \mathrm{Ar}: \mathrm{O}_{2}$ 比例为 $3: 1$; 功率为 $50 \mathrm{~W}$; 溅 射时间为 $4 \mathrm{~h}$ 。

(4)磁控溅射法在 $\mathrm{BFO} / \mathrm{LSCO} / \mathrm{STO}$ 异质结上生 长 $\mathrm{Pt}$ 电极

利用掩膜技术在异质结 $\mathrm{BFO} / \mathrm{LSCO} / \mathrm{STO}$ 上生 长 $\mathrm{Pt}$ 电极, 其面积为 $7.85 \times 10^{-5} \mathrm{~cm}^{2}$, 然后将样品放 入快速退火炉中, 在 $550^{\circ} \mathrm{C}$ 氧气气氛中退火 $1 \mathrm{~min}$ 。

\section{2 样品性能表征}

采用 $\mathrm{AFM}$ (Bruker) 原子力显微镜观察 $\mathrm{BFO}$ 薄膜 表面形貌。采用丹东 TD-3700 型 $\mathrm{X}$ 射线衍射仪 $(\mathrm{Cu}$ $\mathrm{K} \alpha$ 辐射, 管压为 $30 \mathrm{kV}$, 电流为 $20 \mathrm{~mA}$ ) 对样品进行 相结构分析, $2 \theta$ 扫描范围 $20^{\circ} \sim 50^{\circ}$, 步长 $0.02^{\circ}$ 。采用 AgilentE4980 LCR 介电测试仪测试样品的介电性 能。采用 Kiethley2602 多功能数字表对 $\mathrm{Pt} / \mathrm{BFO} /$ LSCO 异质结进行 $I-V$ 测试。 


\section{2 结果与讨论}

\section{$2.1 \mathrm{BFO} / \mathrm{LSCO} / \mathrm{STO}$ 异质结微结构表征}

图 1(a)为 BFO 薄膜的 AFM 表面形貌图, AFM 的扫描模式为接触模式。由图 1(a) 可以看出, 在 $\mathrm{LSCO} / \mathrm{STO}$ 异质结上外延生长的 BFO 薄膜结构致密, 具有层状的表面和均匀细小的晶粒, 表面平均粗粘 度(RMS)仅为 $0.775 \mathrm{~nm}$, 表明 $\mathrm{BFO}$ 薄膜结晶质量较 高。为了分析制备的 BFO 薄膜外延结构, 对 $\mathrm{BFO} /$ $\mathrm{LSCO} / \mathrm{STO}$ 异质结进行了 $\theta-2 \theta$ 的 XRD 图谱扫描, 如图 1(b)所示。由图 1(b)可以看出, 衍射角 $2 \theta$ 在 $20^{\circ} \sim 50^{\circ}$ 扫描范围内仅出现了 LSCO 和 BFO 薄膜的 (001)和(002)两个特征峰, 且与基底 STO $(00 l)$ 晶向 相同，表明在 LSCO 底电极上实现了 BFO 薄膜的外 延生长。(001) BFO 峰较小的半宽高为 0.259 , 从另 一个层面说明 $\mathrm{BFO}$ 薄膜具有良好的结晶质量。为了 进一步确定 BFO 薄膜的外延性, 通过 XRD 对 BFO 薄膜的(110)面进行了 $\Phi$ 扫描, 如图 1(b)插图所示, 图 谱中仅有四个等角相距为 $90^{\circ}$ 的尖锐衍射峰, 说明 $\mathrm{BFO}$ 薄膜是以 $[100]_{\mathrm{BFO}} / /[100]_{\mathrm{LSCO} / \mathrm{STO}}$ 的取向方式异 质外延生长, $\mathrm{BFO}$ 薄膜表面与 $\mathrm{LSCO} / \mathrm{STO}$ 表面呈现 为四重对称性匹配。
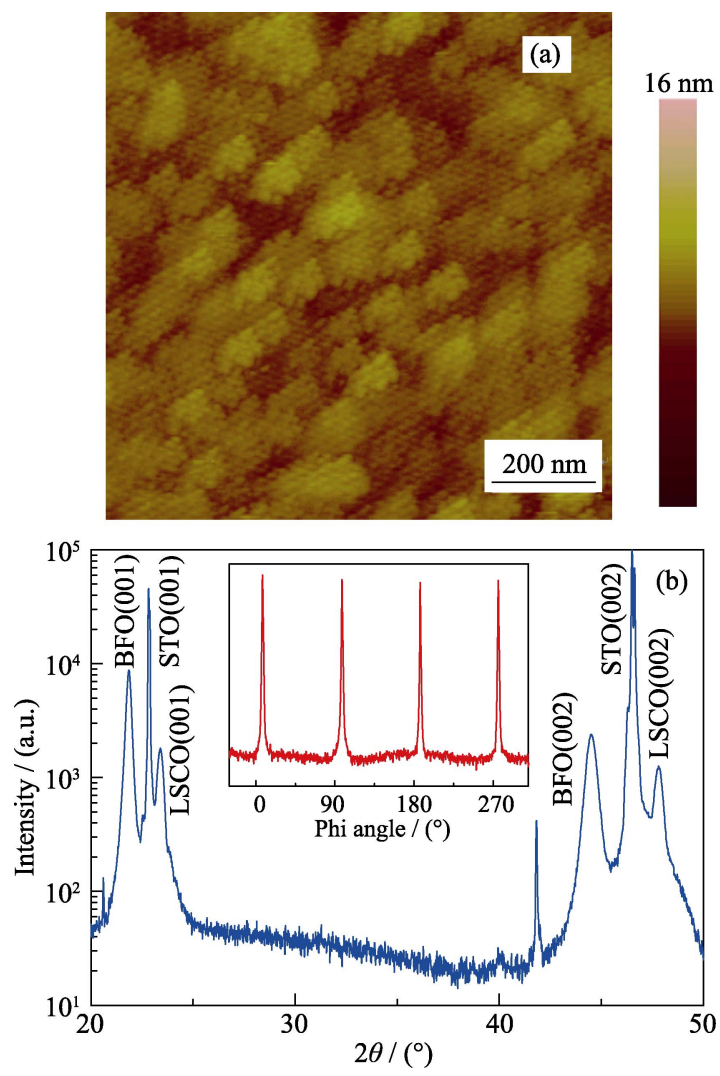

图 1 (a) BFO 薄膜的 AFM 图; (b) BFO/LSCO/STO 异质结的 $X$ 射线衍射图谱

Fig. 1 (a) AFM image of BFO thin film and (b) XRD pattern of $\mathrm{BFO} / \mathrm{LSCO} / \mathrm{STO}$ heterostructures

\section{$2.2 \mathrm{Pt} / \mathrm{BFO} / \mathrm{LSCO} / \mathrm{STO}$ 异质结的介电性能}

介电常数和介电损耗是表征电容器介电性能的 两个重要参数。图 2(a)为 $\mathrm{Pt} / \mathrm{BFO} / \mathrm{LSCO}$ 电容器介电 常数和介电损耗随频率的变化曲线, 由图可以看出, 频率低于 $1 \mathrm{MHz}$ 时, 介电常数随频率的增大而成线 性减小，而介电损耗变化不大，保持在 0.12 ; 当频 率大于 $1 \mathrm{MHz}$ 时, 介电常数下降较快, 且介电损耗 开始增大。文献报道铁电材料的介电性能与异质结

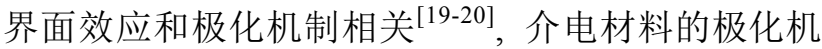
制可以归结为四种：电子极化、离子极化、偶极子极 化和空间电荷极化。频率较低时，由于 $\mathrm{Pt} / \mathrm{BFO} / \mathrm{LSCO}$ 电容器上电极 Pt 易生成氧化层, 等同于电容器串联 了一个电阻, 从而导致介电常数减小, 介电损耗影 响不大。另外, 低频率时, 极子极化翻转可以与外加 信号频率同步，对介电性能影响小，表明低频率时界 面电阻效应为主导作用。当测试频率大于 $1 \mathrm{MHz}$ 时, 随着频率的升高，某些极子的极化跟不上频率的变 化，例如离子比电子对电场的响应慢，而产生极化 弛豫，此时，电阻效应和极子极化弛豫效应共
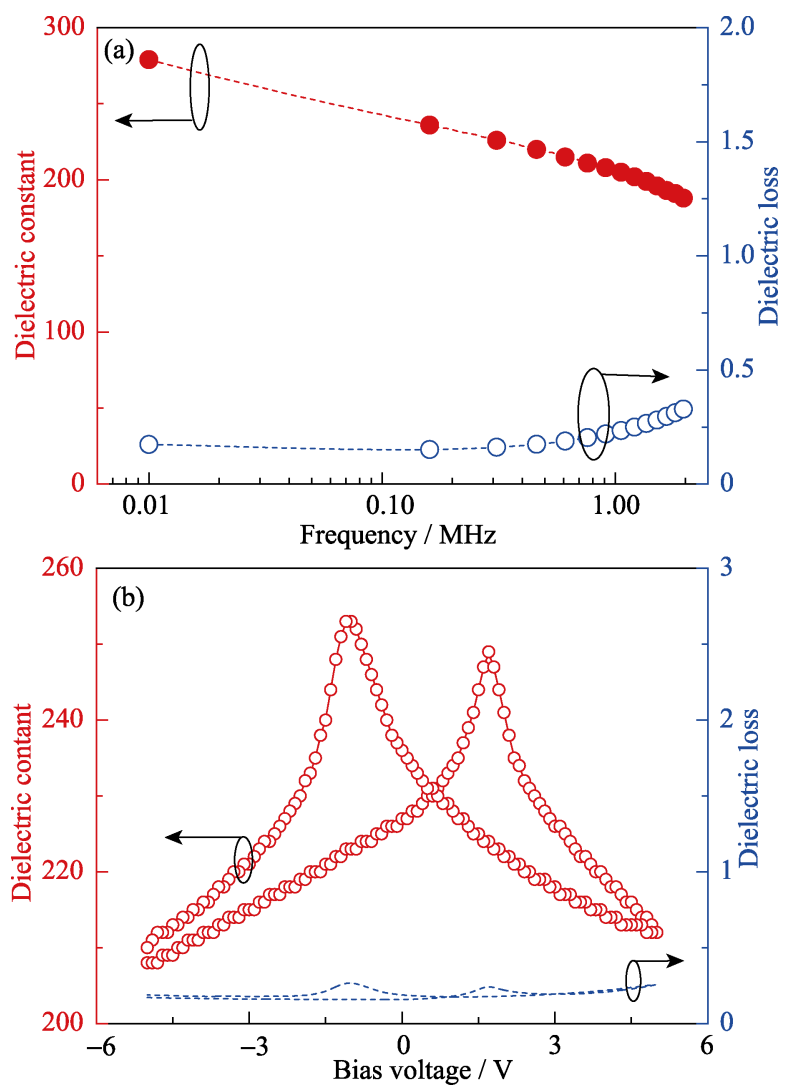

图 2 (a) $\mathrm{Pt} / \mathrm{BFO} / \mathrm{LSCO}$ 异质结中 $\mathrm{BFO}$ 薄膜的介电频谱; (b) $\mathrm{Pt} / \mathrm{BFO} / \mathrm{LSCO}$ 异质结中 $\mathrm{BFO}$ 薄膜的介电常数和介电损耗随 电压的变化曲线

Fig. 2 (a) Frequency dependence of dielectric constant and dielectric loss of BFO thin film in $\mathrm{Pt} / \mathrm{BFO} / \mathrm{LSCO}$ heterostructures, and (b) voltage dependence of dielectric constant and dielectric loss of $\mathrm{BFO}$ thin film in $\mathrm{Pt} / \mathrm{BFO} / \mathrm{LSCO}$ heterostructures 
同作用于 BFO 薄膜, 且极化弛豫效应起主导作用, 从而造成介电常数和介电损耗变化较快。

图 2(b) 为 $\mathrm{Pt} / \mathrm{BFO} / \mathrm{LSCO}$ 电容器的相对介电常数 和介电损耗随电压的变化曲线, 测试采用循环扫描 的方式 $-5 \rightarrow 5 \rightarrow-5 \mathrm{~V}$, 测试频率 $1 \mathrm{MHz}$ 。由图 2(b) 可 以看出, 介电常数与电压的关系呈现典型的饱和蝶 形曲线, 表明 BFO 薄膜具有铁电性能和极化反转特 性。在正负矫顽电压分别为 $1.7 \mathrm{~V}$ 和 $-1.1 \mathrm{~V}$ 时, 介电 常数分别为 249 和 253 , 不具有对称性, 这是由于不 同的 $\mathrm{Pt} / \mathrm{BFO}$ 和 $\mathrm{BFO} / \mathrm{LSCO}$ 界面形成了不同的界面 势垒造成的。在实际应用中, 人们希望得到高调谐 率低耗损的薄膜, 由图 2(b) 可以得出, 在 $5 \mathrm{~V}$ 电压作 用下, $\mathrm{Pt} / \mathrm{BFO} / \mathrm{LSCO}$ 电容器的调谐率和介电损耗分 别为 $14.1 \%$ 和 0.19 , 显示出优异的介电性能。

\section{$2.3 \mathrm{Pt} / \mathrm{BFO} / \mathrm{LSCO} / \mathrm{STO}$ 异质结的阻变机制}

图 3 为 $\mathrm{Pt} / \mathrm{BFO} / \mathrm{LSCO}$ 异质结阻变存储器在 $5 \mathrm{~V}$ 作用下的 $I-V$ 曲线，其中图 3 (a)为线性坐标，图 3 (b) 为对数坐标。由图 3(a) 可知, 在 $0 \rightarrow 5 \rightarrow 0 \mathrm{~V}$ 的电压 扫描中, $\mathrm{Pt} / \mathrm{BFO} / \mathrm{LSCO}$ 异质结阻变器件从高阻态变 为低阻态，而在随后的 $0 \rightarrow-5 \rightarrow 0 \mathrm{~V}$ 的电压扫描中, 阻变器件再次从高阻态转变为低阻态, 未表现出零 偏压时交叉形式的逆时针滞后回线(电致阻变现
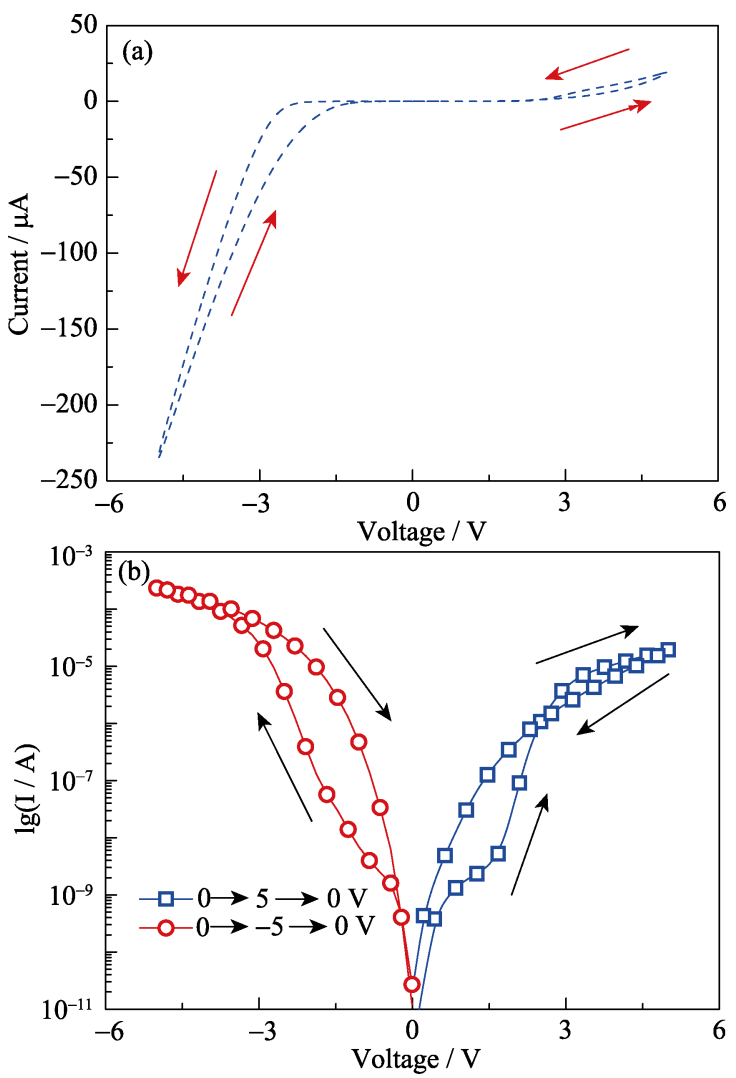

图 $3 \mathrm{Pt} / \mathrm{BFO} / \mathrm{LSCO}$ 异质结 $I-V$ 曲线

Fig. $3 \quad I-V$ curves of $\mathrm{Pt} / \mathrm{BFO} / \mathrm{LSCO}$ heterostructures (a) Linear coordinate; (b) Logarithmic coordinate
象)。此种转变是典型的铁电二极管的阻变开关行 为。另外, 在正向偏压一侧表现出明显的整流效应, 这是由于 $\mathrm{Pt} / \mathrm{BFO}$ 和 $\mathrm{BFO} / \mathrm{LSCO}$ 界面具有不同肖特 基势垒造成的。 $\mathrm{Pt} 、 \mathrm{BFO}$ 和 LSCO 的功函数分别为 5.36、4.7 和 $4.65 \mathrm{eV}^{[8,21]}$ 。根据功函数数据分析可知, 上界面 $\mathrm{Pt} / \mathrm{BFO}$ 肖特基势垒远高于 $\mathrm{BFO} / \mathrm{LSCO}$ 界面。 也就是说，上界面处内建电场较强，正电荷密度较 大, 因此正电压作用下的漏电流要比负电压下小。 对比图 3(a)正负偏压下的曲线可以看出, 阻变窗口 在正负偏压下表现出明显的非对称性，在正向偏压 下回滞窗口较小，负向偏压下回滞窗口较大，图 3(b) 的 $\lg I-V$ 曲线清晰地显示出这种差异。

为了探讨样品的导电机制, 对 $\lg I-\lg V$ 曲线进行 了线性拟合，图 4(a)和(b)分别是正向偏压和负向偏 压下的拟合曲线。图 $4(\mathrm{a})$ 曲线表明, $0 \rightarrow 5 \rightarrow 0 \mathrm{~V}$ 正向 电压作用下，高阻态和低阻态均符合空间电荷限制 电流(SCLC)理论 ${ }^{[22-24]}$, 随着电压的增大, $\lg I$ 随 $\lg V$ 曲线依次遵循: 线性欧姆导电 $(S \sim 1)$ 、平方率导电 $(S \sim 2)$ 以及陷阱能级的填充和脱陷导电 $(S>>2)$, 其中 $S$ 表示曲线斜率，这与其它文献报道的 $\mathrm{Au} / \mathrm{BFO} /$ $\mathrm{SrRuO}_{3}$ 异质结阻变机制相同 ${ }^{[25]}$ 。此外, 由图 4(b)曲 线可以看出, $0 \rightarrow-5 \mathrm{~V}$ 负向电压作用下, $\mathrm{Pt} / \mathrm{BFO} / \mathrm{LSCO}$ 阻变器处于高阻态, 且具有与正向电压相同的阻变
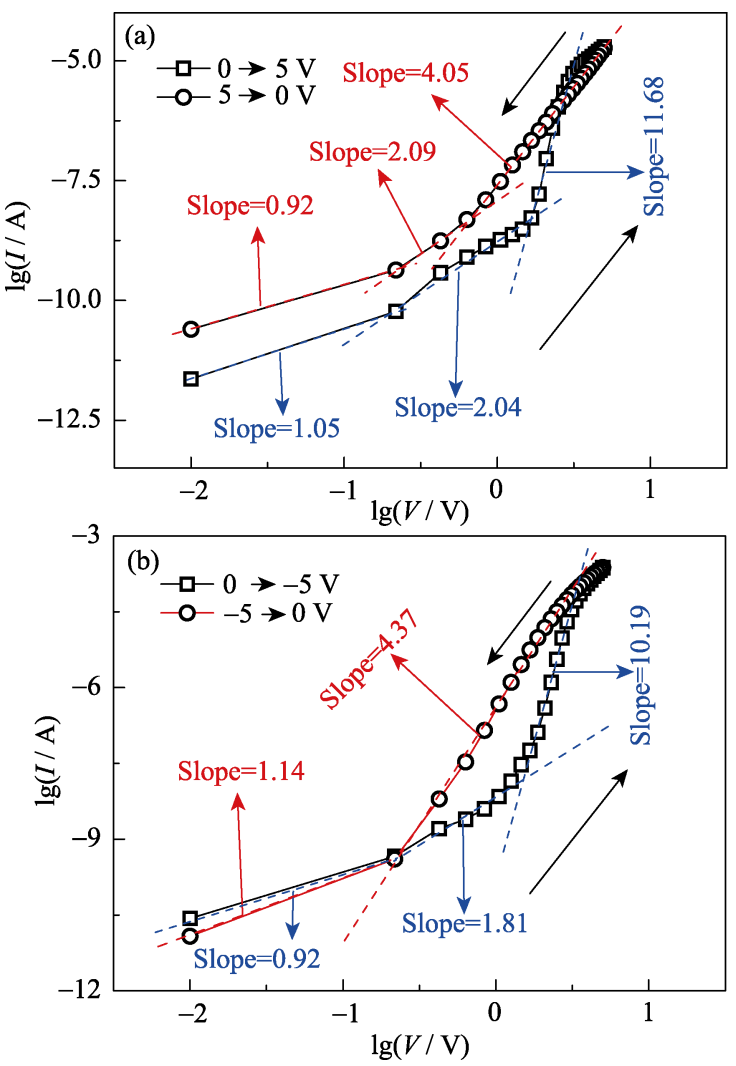

图 $4 \mathrm{Pt} / \mathrm{BFO} / \mathrm{LSCO}$ 异质结 $\lg I-\lg V$ 拟合曲线

Fig. $4 \lg I-\lg V$ fitting curves of $\mathrm{Pt} / \mathrm{BFO} / \mathrm{LSCO}$ heterostructures 
机制，即空间电荷限制电流理论。然而， $-5 \rightarrow 0 \mathrm{~V}$ 负 向电压作用下，低阻态在 SCLC 机制下也具有良好 的线性度, 但高压时斜率 $(S \sim 4)$ 与 SCLC 理论中斜率 $(S \sim 2)$ 不符, 因此, 可以推断 $-5 \rightarrow 0 \mathrm{~V}$ 作用下的低阻 态为其它类型导电机制。图 5 为 $\mathrm{Pt} / \mathrm{BFO} / \mathrm{LSCO}$ 异质 结在 $-5 \rightarrow 0 \mathrm{~V}$ 作用下 $\ln \left(J / V^{2}\right)$ 与 $V^{-1}$ 拟合曲线, $J$ 表示 电流密度, 由图 5 拟合曲线可以看出, 当偏压 $|V|$ 大 于 $0.8 \mathrm{~V}$ 时, $\ln \left(J / V^{2}\right)$ 与 $V^{-1}$ 满足线性关系，表明 $\mathrm{Pt} / \mathrm{BFO} / \mathrm{LSCO}$ 异质结在 $-5 \rightarrow 0 \mathrm{~V}$ 作用下的低阻态由 界面限制的 F-N 隧穿机制主导 ${ }^{[26]}$ 。

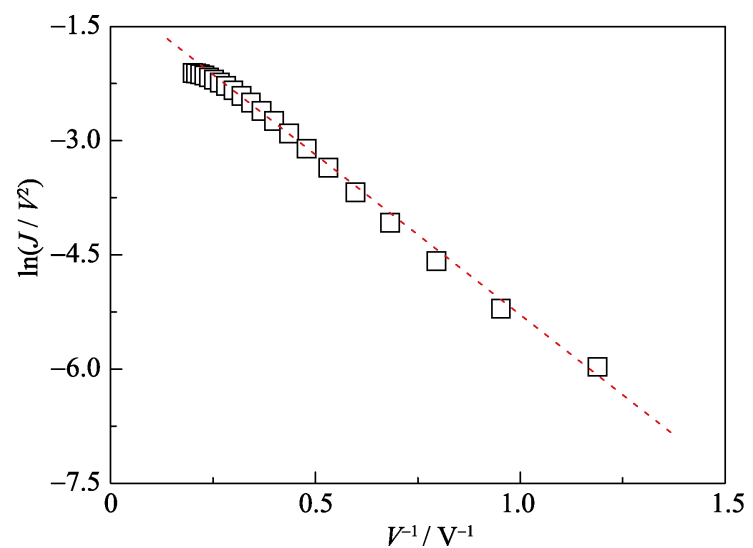

图 $5 \mathrm{Pt} / \mathrm{BFO} / \mathrm{LSCO}$ 异质结 $-5 \rightarrow 0 \mathrm{~V}$ 电压下 $\ln \left(J / V^{2}\right)-V^{-1}$ 拟合 曲线

Fig. $5 \ln \left(J / V^{2}\right)-V^{-1}$ fitting curve of $\mathrm{Pt} / \mathrm{BFO} / \mathrm{LSCO}$ heterostructures at $-5 \mathrm{~V} \rightarrow 0 \mathrm{~V}$

\section{3 结论}

以 LSCO 作为底电极, 利用偏轴射频磁控溅射法 在(001)STO 祄底上成功制备了 $\mathrm{Pt} / \mathrm{BFO} / \mathrm{LSCO} / \mathrm{STO}$ 铁电电容器异质结。AFM 图和 X 射线衍射图谱表 明, BFO 薄膜具有良好的结晶质量, 且沿(001)方向 生长的外延结构。介电性能测试表明, 在 $5 \mathrm{~V}$ 驱动 电压下, 电容器呈现饱和的蝶形回线, 介电常数和 调谐率分别为 253 和 $14.1 \%$ 。阻变机制研究发现, $0 \rightarrow 5 \rightarrow 0 \rightarrow-5 \mathrm{~V}$ 时为空间电荷限制电流陷阱能级的 填充和脱陷, $-5 \rightarrow 0 \mathrm{~V}$ 时符合界面限制的 F-N 隧穿机 制。此实验结果可为无铅铁酸铋铁电阻变存储器件 的开发与研究提供数据参考。

\section{参考文献:}

[1] WEN Z, LI C, WU D, et al. Ferroelectric-field-effect-enhanced electroresistance in metal/ferroelectric/semiconductor tunnel junctions. Nature Mater., 2013, 12(7): 617-621.

[2] TIAN B B, LIU Y, CHEN L F, et al. Space-charge effect on electroresistance in metal-ferroelectric-metal capacitors. Sci. Rep., 2015, 5: 18297-1-9.

[3] ABUWASIB M, LU H, LI T, et al. Scaling of electroresistance effect in fully integrated ferroelectric tunnel junctions. Appl. Phys.
Lett., 2016, 108(15): 152904-1-5.

[4] SILVA J P B, KAMAKSHI K, SEKHAR K C, et al. Light-controlled resistive switching in laser-assisted annealed $\mathrm{Ba}_{0.8} \mathrm{Sr}_{0.2} \mathrm{TiO}_{3}$ thin films. Physica. Status. Solidi. A, 2016, 213(4): 1082-1087.

[5] BARRIONUEVO D, ZHANG L, ORTEGA N, et al. Enhanced tunneling electroresistance in Pt/PZT/LSMO ferroelectric tunnel junctions in presence of magnetic field. Integr. Ferroelectr., 2016, 174(1): $174-185$.

[6] WANG C, JIN K J, XU Z T, et al. Switchable diode effect and ferroelectric resistive switching in epitaxial $\mathrm{BiFeO}_{3}$ thin films. Appl. Phys. Lett., 2011, 98(19): 192901-1-3.

[7] XU H M, WANG H C, SHEN Y, et al. Photocatalytic and magnetic behaviors of $\mathrm{BiFeO}_{3}$ thin films deposited on different substrates. J. Appl. Phys., 2014, 116(17): 174307-1-5.

[8] MOUBAH R, ROUSSEAU O, COLSON D, et al. Photoelectric effects in single domain $\mathrm{BiFeO}_{3}$ crystals. Adv. Funct. Mater., 2012, 22(22): 4814-4818.

[9] LI M, ZHUGE F, ZHU X, et al. Nonvolatile resistive switching in metal/La-doped $\mathrm{BiFeO}_{3} / \mathrm{Pt}$ sandwiches. Nanotech., 2010, 21(42): $425202-1-5$

[10] ZHU H, ZHANG Y, JIANG A, et al. Effect of poling process on resistive switching in $\mathrm{Au} / \mathrm{BiFeO}_{3} / \mathrm{SrRuO}_{3}$ structures. Appl. Phys. Lett., 2016, 109(25): 252901-1-4.

[11] HONG S, CHOI T, JEON J H, et al. Large resistive switching in ferroelectric $\mathrm{BiFeO}_{3}$ nano-island based switchable diodes. Adv. Mater, 2013, 25(16): 2339-2343.

[12] YAMADA H, GARCIA V, FUSIL S, et al. Giant electroresistance of super-tetragonal $\mathrm{BiFeO}_{3}$-based ferroelectric tunnel junctions. ACS Nano., 2013, 7(6): 5385-5390.

[13] LIN Y B, YAN Z B, LU X B, et al. Temperature-dependent and polarization-tuned resistive switching in $\mathrm{Au} / \mathrm{BiFeO} / \mathrm{SrRuO}_{3}$ junctions. Appl. Phys. Lett., 2014, 104(14): 143503-1-5.

[14] WANG Y, JIANG Q H, HE H C, et al. Multiferroic $\mathrm{BiFeO}_{3}$ thin films prepared via a simple Sol-Gel method. Appl. Phys. Lett., 2006, 88(14): 142503-1-3.

[15] TRASSIN M, CLARKSON J D, BOWDEN S R, et al. Interfacial coupling in multiferroic-ferromagnet heterostructures. Phys. Rev. B, 2013, 87(13): 134426-1-6.

[16] QI X, TSAI PC, CHEN YC, et al. Ferroelectric properties and dielectric responses of multiferroic $\mathrm{BiFeO}_{3}$ films grown by $\mathrm{RF}$ magnetron sputtering. J. Phys. D: Appl. Phys., 2008, 41(23): 232001-1-5.

[17] WU J, WANG J. $\mathrm{BiFeO}_{3}$ thin films of (111)-orientation deposited on $\mathrm{SrRuO}_{3}$ buffered $\mathrm{Pt} / \mathrm{TiO} / \mathrm{SiO} / \mathrm{Si}(100)$ substrates. Acta Mater. 2010, 58(5): 1688-1697.

[18] HAO Y L, LIU B T, PENG Z W, et al. Effect of retained temperature on the structure and property of $\mathrm{BiFeO}_{3}$ thin film prepared by off-axis magnetron sputtering method. J. Synth. Cryst., 2013, 42(2): 246-250

[19] FAN X M, LIAN J S, GUO Z X, et al. Microstructure and photoluminescence properties of $\mathrm{ZnO}$ thin films grown by PLD on Si(111) substrates. Appl. Surf. Sci., 2005, 239(2): 176-181.

[20] WANG C, CHEN Z, HU H, et al. Effect of the oxygen pressure on the microstructure and optical properties of $\mathrm{ZnO}$ films prepared by laser molecular beam epitaxy. Phys. B, 2009, 404(21): 4075-4082.

[21] NAGARAJ B, AGGARWAL S, RAMESH R. Influence of contact electrodes on leakage characteristics in ferroelectric thin films. $J$. Appl. Phys., 2001, 90(1): 375-382.

[22] SHANG D S, WANG Q, CHEN L D, et al. Effect of carrier trapping on the hysteretic current-voltage characteristics in $\mathrm{Ag} / \mathrm{La} \mathrm{a}_{0.7} \mathrm{Ca}_{0.3}$ $\mathrm{MnO}_{3} / \mathrm{Pt}$ heterostructures. Phys. Rev. B, 2006, 73(24): 245427-1-7.

[23] WANG S Y, CHENG B L, WANG C, et al. Reduction of leakage current by Co doping in $\mathrm{Pt} / \mathrm{Ba}_{0.5} \mathrm{Sr}_{0.5} \mathrm{TiO}_{3} / \mathrm{Nb}-\mathrm{SrTiO}_{3}$ capacitor. Appl. Phys. Lett., 2004, 84(20): 4116-4118.

[24] CHEN X, WU G, JIANG P, et al. Colossal resistance switching effect in $\mathrm{Pt} /$ spinel-MgZnO/Pt devices for nonvolatile memory applications. Appl. Phys. Lett., 2009, 94(3): 033501-1-3.

[25] ZHU H, ZHANG Y Q, WANG P F, et al. Resistive switching effect and conduction mechanism of $\mathrm{BiFeO}_{3}$ thin films. J. Chin. Ceram. Soc., 2017, 45(4): 467-471.

[26] EBRAHIMPOUR Z, MANSOUR N. Annealing effects on electrical behavior of gold nanoparticle film: conversion of ohmic to non-ohmic conductivity. Appl. Surf. Sci., 2017, 394: 240-247. 